\title{
Taste/taste potentiation as a function of age and stimulus intensity
}

\author{
STEPHEN F. DAVIS and SCOTT A. BAILEY \\ Emporia State University, Emporia, Kansas \\ and \\ ANGELA H. BECKER and CATHY A. GROVER \\ Texas A\&M University, College Station, Texas
}

\begin{abstract}
Bouton, Dunlap, and Swartzentruber (1987) and Davis, Best, and Grover (1988) have shown that the aversion conditioned to a target taste can be enhanced or potentiated by the presence of a second taste during the conditioning episode. In the present experiment, groups of young (80-day-old) and old (150-day-old) rats were exposed to a CS composed of one or two tastes, respectively, prior to lithium-induced toxicosis. The results indicated that taste potentiation was shown by the younger subjects only when weaker fluid concentrations were employed. However, the effect was shown by the older animals under both weak and strong concentrations.
\end{abstract}

Recent reports by Bouton, Dunlap, and Swartzentruber (1987) and Davis, Best, and Grover (1988) have demonstrated that aversive conditioning of an ingestional compound composed of two tastes can result in potentiated (enhanced) conditioning of one of the tastes. More specifically, Davis, Best, and Grover reported that denatonium saccharide potentiated the aversion to sodium saccharin when exposure to the compound formed by these two tastes was followed by an injection of lithium chloride $(\mathrm{LiCl})$. Moreover, it was found that the presentation of these elements sequentially during conditioning, as well as the extinction of one of the elements following conditioning, eliminated the potentiation effect. These results were interpreted as reflecting the importance of associations formed between the two elements of the compound conditional stimulus (CS) in the mediation of this potentiation effect.

When subsequent experiments using younger subjects failed to produce this potentiation effect (Davis, Erickson, Hoskinson, \& Wilder, 1987), or demonstrated it only when a weaker solution was employed (Best et al., 1989), a more thorough examination of the relevant parameters was undertaken. The present experiment involved the simultaneous evaluation of two of these parameters-age and fluid concentration. More specifically, young (80-dayold) and old (150-day-old) rats were administered $\mathrm{LiCl}$ following exposure to weak solutions of saccharin or a mixture of saccharin and denatonium saccharide (denatonium). Comparable sets of young and old animals also were administered $\mathrm{LiCl}$ following exposure to stronger concentrations of these fluids.

Correspondence may be addressed to Stephen F. Davis, Department of Psychology, Emporia State University, Emporia, KS 66801.

\section{METHOD}

\section{Subjects and Apparatus}

A total of 80 male Holtzman rats (40 80-day-old, 40 150-day-old) served as subjects. All subjects were housed in individual, suspended wire-mesh cages in the animal vivarium. Food was available on a freefeeding basis for the duration of the experiment. All testing was conducted in the home cage.

\section{Procedure}

Prior to the start of the experiment, four equal-sized $(n=10)$ groups (WS = weak saccharin; $\mathbf{W M}=$ weak mix; SS = strong saccharin; SM = strong $\mathrm{mix}$ ) were formed within each age category. These group designations referred to: (1) a $0.075 \%$ (w/v) saccharin solution (WS), (2) a $0.15 \%(w / v)$ saccharin solution (SS), (3) the mixture of weak saccharin and 1 part denatonium saccharide per 20,000 parts water (WM), and (4) the mixture of strong saccharin and 1 part denatonium saccharide per 10,000 parts water (SM).

The experiment encompassed 12 days: baseline (10 days), conditioning ( 1 day), and preference test ( 1 day). Experimental sessions, during which fluids were presented in 50-ml centrifuge tubes with spill-resistant sippers, were conducted at $0800 \mathrm{~h}$. Supplemental water was provided for each animal for $10 \mathrm{~min}$ at $1400 \mathrm{~h}$.

The water-consumption baseline was established by allowing each subject 10 -min access to water daily for the first 10 days of the experiment. Conditioning consisted of a 10-min exposure to the designated fluid, either saccharin (SAC) or a saccharin-denatonium saccharide mixture (MIX). Five minutes later, an intraperitoneal injection of $\mathrm{LiCl}$ $(12 \mathrm{mg} / \mathrm{kg}$ of $0.30 \mathrm{M})$ was administered to each subject. The two-bottle preference test, conducted $24 \mathrm{~h}$ later, consisted of 10 -min access to plain tap water and the appropriate saccharin solution (weak or strong) that had been experienced during conditioning.

\section{RESULTS AND DISCUSSION}

Because analysis of variance failed to yield significance for any effect on the last 2 days of baseline [groups, $F(3,36)=1.13$; days, $F(1,36)=0.96]$ and on the conditioning day [groups, $F(3,36)=0.67$ ], intake equivalence was established prior to the preference test. The 


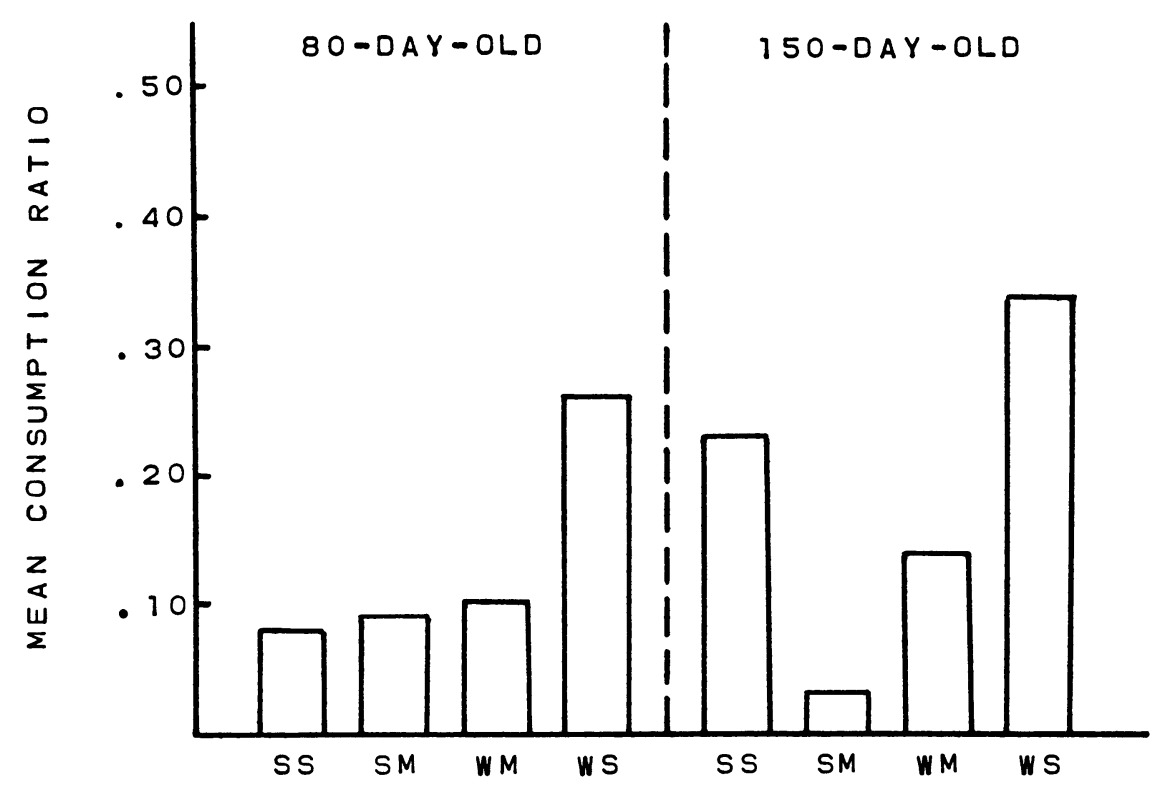

GROUPS

Figure 1. Group mean consumption scores (SAC/SAC + water) for the strong SAC (ȘS), strong MIX (SM), weak MIX (WM), and weak SAC (WS) groups as a function of age.

results of the two-bottle preference test are shown in Figure 1. The consumption ratios shown in this figure were calculated by dividing the amount of saccharin consumed (in $\mathrm{ml}$ ) by the total amount of fluid consumed (saccharin + water). Hence, a ratio of .50 would reflect equal consumption of saccharin and water, whereas ratios lower than .50 would reflect a saccharin aversion.

An analysis of variance incorporating age (80- vs. 150day-old animals), concentration (weak vs. strong), and type of CS (single- vs. multiple-element) as main effects was performed on the consumption-ratio data from the preference test. The results of this analysis yielded significance for the main effects of concentration $[F(1,72)$ $=5.27, p<.05]$ and type of $\operatorname{CS}[F(1,72)=7.19$, $p<.01]$. Moreover, the concentration $\times$ type of CS $[F(1,72)=8.03, p<.01]$, age $\times$ type of CS $[F(1,72)$ $=7.66, p<.01]$, and age $\times$ concentration $\times$ type of $\operatorname{CS}[F(1,72)=8.87, p<.01]$ effects also achieved significance.

Simple main-effects analyses were employed to systematically probe the significant three-way interaction. For the 80-day-old animals, comparisons of consumption ratios for the single- and multiple-element groups yielded significance only when the weak concentrations were involved [WS vs. WM, $F(1,72)=6.33, p<.05$ ]. However, comparisons of the type of CS for the 150-dayold animals yielded significance for both the strong $[F(1,72)=9.56, p<.01]$ and the weak $[F(1,72)=7.74$, $p<.01]$ concentrations. Subsequent Newman-Keuls tests applied to the data from the 150-day-old animals indicated that the consumption ratios of Group SM were signifi- cantly $(p<.01)$ lower than those of Groups SS, WM, and WS. Group WM was significantly $(p<.05)$ lower than Groups SS and WS, and Group SS was significantly $(p<.05)$ lower than Group WS.

These data replicate those reported by Bouton et al. (1987) and Davis et al. (1988). Clearly, strong potentiation effects were shown by both the 80- and the 150-dayold animals. However, for the 80-day-old animals, this effect was shown only by the subjects that were conditioned to the weak solution (i.e., the aversion of Group WM was significantly stronger than that of Group WS). This outcome points to ontogenetic changes in the sensitivity to taste combinations and may reflect the importance of the salience balance between ingestional stimuli in the production of potentiation (see Bouton et al., 1987). In this regard, it is possible that the strong mix in the 80-day-old animals was too intense to provide the necessary stimulus balance for the production of potentiation. This is of particular interest in light of the relative ease with which taste-mediated potentiation can be produced in young as opposed to older rats (e.g., Kucharski \& Spear, 1985; Peterson, Valliere, Misanin, \& Hinderliter, 1985).

\section{REFERENCES}

Best, M. R., Davis, S. F., LoOK, C. T., Becker, A. H., Syler, J. C., \& NeAL, L. (1989, April). Taste/taste potentiation in the young rat: Effect of CS-US interval. Paper presented at the annual meeting of the Southwestern Psychological Association, Houston.

Bouton, M. E., Dunlap, C. M., \& Swartzentruber, D. (1987). Potentiation of taste by another taste during compound aversion conditioning. Animal Learning \& Behavior, 15, 433-438. 
Davis, S. F., Best, M. R., \& Grover, C. A. (1988). Toxicosis-mediated potentiation in a taste/taste compound: Evidence for within-compound associations. Learning \& Motivation, 19, 183-205.

Davis, S. F., Erickson, C. A., Hoskinson, K., \& Wilder, K. (1987). Potentiation of a conditioned taste aversion as a function of CS intensity in a taste/taste compound. Paper presented at the annual meeting of the Association for Psychological and Educational Research in Kansas, Lawrence, KS.
KuCharSKI, D., \& SPAR, N. E. (1985). Potentiation and overshadowing in preweanling and adult rats. Journal of Experimental Psychology: Animal Behavior Processes, 11, 15-34.

Peterson, C. S., Valliere, W. A., Misanin, J. R., \& Hinderliter, C. F. (1985). Age differences in the potentiation of taste aversion by odor cues. Physiological Psychology, 13, 103-106.

(Manuscript received September 30, 1989.) 